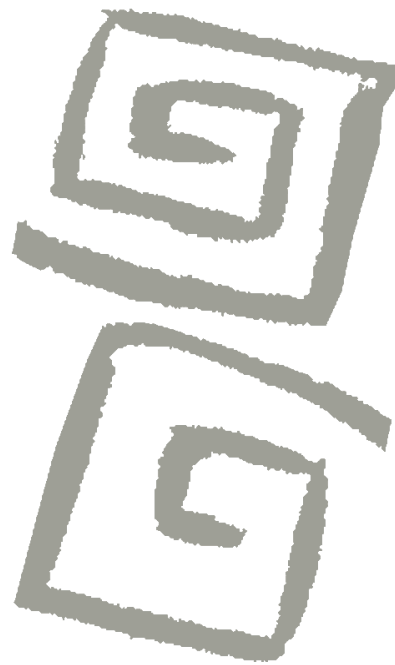

\title{
El universalismo en salud en Argentina entre 2003 y 2015: balances y desafíos desde una aproximación macroinstitucional
}

\author{
Health universalism in Argentina between 2003 and \\ 2015: assessments and challenges based in a macro- \\ institutional approach
}

Magdalena Chiara ${ }^{1}$, María Crojethovic ${ }^{2}$, Ana Ariovich ${ }^{3}$

${ }^{1}$ Doctora en Ciencias Sociales. Profesora

Asociada, Instituto del Conurbano, Universidad Nacional de General Sarmiento, Buenos Aires, Argentina. $\triangle$ iD

2 Doctora en Ciencias Sociales. Profesora Adjunta, Instituto del Conurbano, Universidad Nacional de General Sarmiento, Buenos Aires, Argentina. $\triangle$ iD

${ }^{3}$ Magíster en Sociología Económica. Asistente principal, Instituto del Conurbano, Universidad Nacional de General Sarmiento, Buenos Aires, Argentina. $\triangle$ id
RESUMEN Los debates en torno al universalismo en salud fueron ganando terreno en la región e ingresando a la agenda de las políticas con resultados desiguales. Aun en el contexto del federalismo, los cambios más profundos que se sucedieron en Argentina en la última década tuvieron lugar en la arena de la política nacional. A partir de los aportes teóricos del neoinstitucionalismo histórico y de los estudios de implementación, este trabajo se propone analizar el alcance de la política nacional en torno a la universalización en salud, en un nivel macroinstitucional. Se trata de un estudio descriptivo, basado en fuentes secundarias y en la revisión de resultados de investigaciones sobre la implementación del Programa Remediar, el Plan Nacer y el Programa Sumar en relación con cuatro variables: acceso, cobertura, conjunto de beneficios y derechos que recoge la política. Dadas las características de la matriz institucional argentina, puede esperarse que la implementación en los escenarios subnacionales recorra espacios complejos y heterogéneos en los que los programas pueden adquirir nuevos significados con relación al horizonte de la universalidad que cada uno de ellos plantea.

PALABRAS CLAVES Implementación de Plan de Salud; Acceso Universal a Servicios de Salud; Cobertura de Servicios Públicos de Salud; Descentralización; Federalismo; Argentina.

\begin{abstract}
Debates about universalism in health have been gaining ground in Latin America and have entered the policy agenda with differing results. Notwithstanding the country's federalism, the most profound changes that took place in Argentina in the last decade occurred in the arena of national politics. Based on the theoretical contributions of historical neo-institutionalism and implementation studies, this paper aims to analyze, from a macro institutional approach, the scope of the national policy regarding health universalization. This descriptive study is based on secondary sources and the review of research results on the implementation of the programs Remediar, Sumar and Plan Nacer in relation to four variables: coverage, access, sets of benefits and rights included in the policy. Given the characteristics of the Argentine institutional matrix, program implementation in subnational scenarios can be expected to confront complex and heterogeneous terrain in which the programs acquire new meanings with respect to the goal of universality that each poses.
\end{abstract}

KEY WORDS Health Plan Implementation; Universal Access to Health Care Services; State Health Care Coverage; Decentralization; Federalism; Argentina. 


\section{INTRODUCCIÓN}

La cuestión del universalismo ha ganado terreno en la región, tanto en la esfera académica como en la vinculada con la gestión de las políticas de salud; la temática ingresó en la agenda a comienzos del siglo XXI con la propuesta de la Cobertura Universal de Salud promovida por la Organización Mundial de la Salud (OMS) y la Organización Panamericana de la Salud (OPS). Este horizonte abre distintos desafíos al interior de cada país, con relación a los modos a través de los cuales promover la universalidad en salud.

En un estudio previo se identificaron cuatro grandes planos en los que se tensiona el debate. Un primer plano alude al significado que disputa el derecho de los servicios universales, en tanto se trate de una garantía individual o bien de un derecho social que contribuye a la construcción de ciudadanía ${ }^{(1,2)}$. Un segundo plano, refiere a cómo se define el alcance en la universalización, dado que los programas con población objetivo o prestaciones determinadas reconocen un universo social que puede restringir el derecho solo a grupos excluidos( ${ }^{(3)}$. El tercero remite al tipo de prestaciones y servicios que se priorizan, y a cómo y quién define el acceso a dichas prestaciones ${ }^{(4)}$. El último plano en el que se tensiona el debate acerca del universalismo en salud refiere a la capacidad institucional con relación a los recursos, las organizaciones responsables y las capacidades públicas, abordando también los cambios requeridos en el financiamiento y en la gestión de las instituciones que formulan y ejecutan las políticas ${ }^{(5,6)}$.

Acotando la mirada al campo de los servicios, la OMS identificó tres dimensiones para analizar la cobertura universal en salud: servicios, cobertura y financiamiento, este último entendido como la protección económica de la población para no correr el riesgo de empobrecimiento frente al tratamiento de una enfermedad ${ }^{(7)}$. Hacia el año 2014, con el énfasis puesto en la ampliación en el acceso a los servicios para los grupos en situación de vulnerabilidad, la propuesta de la cobertura universal en salud se transformó en el acceso y cobertura universal en salud(8).

El significado que guarda en esta propuesta la universalidad parece estar orientado más hacia el objetivo de la cobertura de población vulnerable o en riesgo, que a garantizar derechos sociales; esta distinción es relevante en tanto restringe el debate al abordaje de la población en riesgo o en situación de vulnerabilidad ${ }^{(6)}$. En este marco, el objetivo de este trabajo es realizar un balance acerca de los alcances hacia la universalización de la política de salud implementada en la Argentina durante la última década haciendo foco en dos dispositivos en particular: el Programa Remediar y el Plan Nacer, convertido posteriormente en el Programa Sumar.

\section{APROXIMACIÓN TEÓRICO- METODOLÓGICA}

Esta investigación recupera los aportes de la perspectiva de la implementación para abordar los recorridos de las políticas hacia el universalismo en salud. Desde este enfoque, la implementación de una política es un proceso complejo que involucra un conjunto de acciones que transforman las intenciones y objetivos iniciales en resultados observables ${ }^{(9)}$; asimismo, la implementación posee un carácter intrínsecamente político que supone la participación de actores con diversos intereses y puntos de vista que deben encontrar arreglos de cooperación para llevar adelante objetivos comunes ${ }^{(10)}$.

Por otra parte, desde diversas aproximaciones, el neo institucionalismo histórico ha postulado que las instituciones influyen en la manera en que los actores de la política forman sus preferencias y definen sus intereses y objetivos, incidiendo -a su vez- en el desenlace de tal política ${ }^{(11)}$. Dentro de esta escuela, el marco institucional comprende desde las reglas de juegos formales e informales que estructuran las relaciones entre grupos o individuos hasta las rutinas organizacionales que se ponen en juego a nivel de 
las prácticas ${ }^{(12,13)}$. En línea con estas contribuciones teóricas, los estudios inscriptos en el denominado "nuevo institucionalismo histórico" refieren, además, a las instituciones como rasgos relativamente persistentes $y$ como factores centrales que empujan el desarrollo histórico a lo largo de un conjunto de "senderos" que condicionan las políticas futuras ${ }^{(14)}$.

Partiendo entonces de este supuesto, el análisis se inicia con una caracterización de la matriz institucional, por cuyos caminos se desenvuelve la política nacional. Con tal propósito, se describen los principales rasgos que definen al sistema político y al sistema de salud en Argentina.

Se trata de un estudio descriptivo a nivel macroinstitucional basado en fuentes secundarias, y en la revisión de resultados de investigaciones para detectar posibles desafíos en la implementación de políticas orientadas a la universalidad en el contexto de la matriz institucional argentina. Una aproximación explicativa a estos fenómenos requeriría no solo un desarrollo mayor, sino delimitar un objeto empírico en el nivel subnacional (a nivel provincial $y / o$ municipal) y formular preguntas a ser respondidas -también y muy especialmente- a partir de fuentes primarias, para recuperar la perspectiva de los actores en juego y sus intereses. Este trabajo es un resultado parcial de la fase inicial del proyecto de investigación titulado "Cobertura universal de salud y redes de servicios: encuentros y desencuentros entre la macro y la meso gestión: Un estudio de caso en el conurbano bonaerense 2008-2015".

Como unidad de análisis de la política de salud en el nivel nacional, orientada a los establecimientos de dependencia estatal, se seleccionaron dos programas o dispositivos que contemplan las siguientes características:

a. Se encuentran bajo la órbita nacional (permiten problematizar las relaciones entre niveles de gobierno).

b. Trascienden temporalmente al menos un período de gobierno.

c. Explicitan en sus documentos institucionales una intención de universalidad. d. Presentan un posicionamiento sectorial que les otorga cierta visibilidad en la esfera pública.

Las orientaciones y los avances hacia el universalismo de los dispositivos de la política sanitaria escogidos fueron estudiados considerando cuatro grandes variables que recuperan los acuerdos teóricos planteados en los principales debates en torno al universalismo, presentados en la introducción de este trabajo:

- Acceso (considerando las condiciones que lo facilitan y la gratuidad en la atención).

- Cobertura (en términos del alcance poblacional).

- Beneficios (prestaciones brindadas en cantidad y calidad).

- Derechos (haciendo referencia al tipo de derecho o derechos que recoge la política).

Desde una perspectiva metodológica, estas variables permiten operacionalizar en la investigación un concepto complejo como el universalismo en el ámbito de la salud, a la vez que hacen posible reconstruir y analizar la política pública en salud acotada a las instituciones pertenecientes al subsector estatal. Aun con las limitaciones que implica, la delimitación del objeto de análisis adoptada en la investigación de la que se deriva este trabajo está fundada en el objetivo que se plantean -al menos en su normativa- las instituciones del subsector estatal: garantizar la atención a la salud de la población independientemente de la condición de la persona que asista a sus establecimientos. Entre las fuentes utilizadas en el relevamiento de los datos secundarios pueden mencionarse: documentos de programas seleccionados, información sobre su cobertura e impacto (informes de avance y publicaciones), leyes nacionales y provinciales, decretos nacionales y provinciales, ordenanzas, convenios para la implementación de los programas, datos epidemiológicos e informes de gestión de los programas de salud analizados.

Finalmente, es importante señalar que las orientaciones elaboradas por organismos 
internacionales para el monitoreo del cumplimiento de los pactos que involucran las garantías del derecho a la salud ${ }^{(15)}$ plantean una serie de estándares óptimos que vinculan estrechamente la universalidad con el derecho a la salud, refiriendo a dimensiones interrelacionadas como la disponibilidad, accesibilidad, aceptabilidad y calidad para ponderar las trayectorias y los desempeños del sector salud en distintos países de la región; abordajes de este tipo requieren, sin embargo, estudios más amplios, que involucren otro tipo de unidades de análisis (como los servicios de salud) y enfoques y herramientas cualitativas en la elaboración de la evidencia empírica.

\section{RESULTADOS}

La organización federal del país consagró una estructura estatal que combina el autogobierno de las provincias con el gobierno compartido que, en su práctica, exige conciliar la unidad nacional del Estado con la autonomía política interna de cada una de ellas ${ }^{(16)}$.

El sector salud en la Argentina está compuesto por tres subsectores (estatal, seguridad social, privado), y es heredero de dos modelos de salud: el de Beveridge, que promueve un sistema nacional y, el de Bismarck, que otorga centralidad a los seguros sociales. El sector se encuentra asentado sobre la provisión pública a la que tienen derecho todos los habitantes con independencia de que dispongan de algún tipo de aseguramiento social o privado. El financiamiento del gasto público total en salud representa un 6,21\% del producto bruto interno (PBI) $)^{(17)}$, con un PBI per cápita calculado para el año 2015 de $\$ 13.431,9$ dólares $^{(18)}$.

Debido al carácter federal, el subsector estatal se organiza en tres niveles de gobierno (nacional, provincial y municipal, en algunas provincias) y ofrece servicios y prestaciones a toda la población. Los trabajadores privados que se desempeñan en el mercado formal y los del sector estatal nacional tienen una cobertura adicional provista por las instituciones de la seguridad social denominadas obras sociales; a su vez, los trabajadores que se desempeñan en los organismos públicos provinciales están asegurados en las obras sociales provinciales. La Figura 1 muestra la distribución porcentual de la población según el tipo de cobertura médico-asistencial ${ }^{(19)}$. Por último, el sector privado está conformado, principalmente, por los prestadores de servicios médico asistenciales, financiadores o empresas de seguros médicos, laboratorios de especialidades médicas, empresas de equipamiento e insumos médicos.

\section{El Programa Remediar, el Plan Nacer y el Programa Sumar}

La década que siguió a la crisis de principio de siglo en Argentina fue testigo de cambios importantes en las ideas acerca del

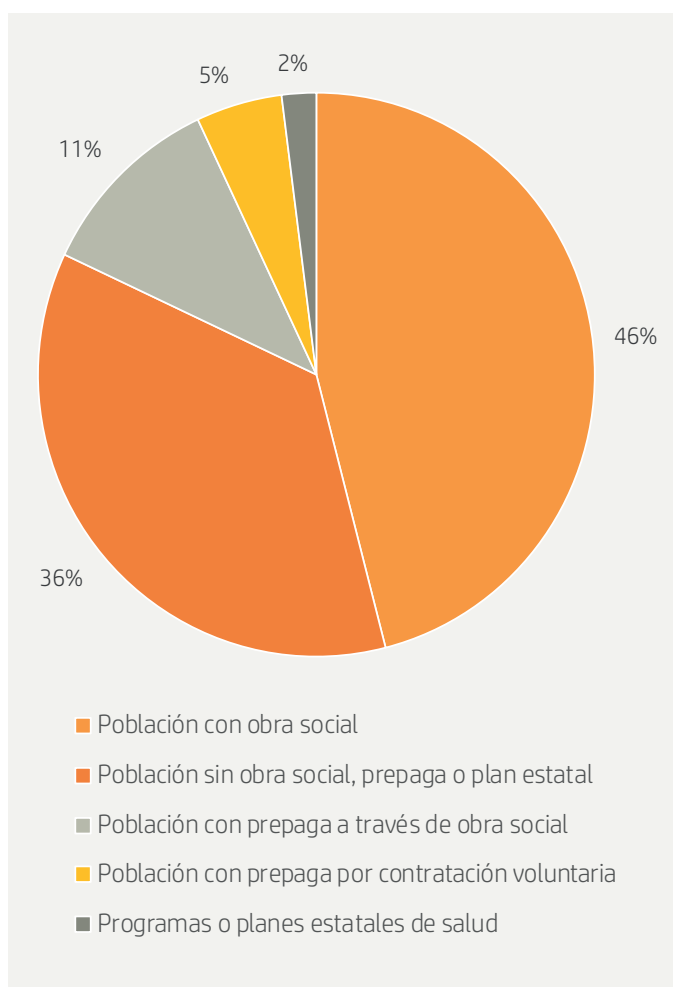

Figura 1. Distribución porcentual de la población ( $N=40.117 .096)$, según cobertura médicoasistencial. Argentina, 2010.

Fuente: Elaboración propia basada en los últimos datos disponibles de población y cobertura en salud del año 2010 del INDEC(19). 
papel del Estado con relación a lo social; distintas medidas muestran la decisión de intervenir sobre las condiciones bajo las cuales se reproduce la vida de la población. De modo silencioso y menos visible, "salud" fue también escenario de cambios; distintos trabajos dan cuenta del surgimiento de iniciativas y de la profundización de otras precedentes $^{(20,21,22,23,24)}$.

La política nacional fue la más sensible a estos procesos. La diferencia más importante respecto de las reformas neoliberales, que caracterizaron a la década anterior, radica en los propósitos que orientaron las propuestas y el regreso del Ministerio de Salud de la Nación como actor a la arena de los servicios, casi en su totalidad bajo la órbita del Estado a nivel provincial y municipal.

Sin alcanzar a ser una reforma de conjunto para el sector, la jerarquización del primer nivel asistencial desde la estrategia de atención primaria de la salud (APS) como organizador del subsector estatal, fue planteada originalmente por el Plan Federal de Salud 2004-2007 y se tradujo en distintos programas que tuvieron a los centros de atención primaria de la salud (CAPS) como instancia privilegiada de ejecución.

Entre las iniciativas más importantes se destacan el Programa Remediar (en sus dos ediciones); el Programa Médicos Comunitarios (con variaciones a lo largo del tiempo); el Programa de Salud Sexual y Procreación Res- ponsable; el Programa Nacional de Control de Tuberculosis y Lepra 2014 (ex Programa Nacional de Control de TBC); el Plan para la reducción de la mortalidad materno infantil, de las mujeres y de las adolescentes; el Programa Nacional de Cardiopatías Congénitas; y el Plan Nacer y el Programa Sumar ${ }^{(20,25,26)}$.

Del conjunto de estas iniciativas nacionales, para esta investigación se seleccionaron dos dispositivos que responden a las características ya definidas para las unidades de análisis: el Programa Remediar, el Plan Nacer y el Programa Sumar (Cuadro 1).

\section{Programa Remediar}

En el marco más amplio de una política de medicamentos (que incluyó su prescripción por el nombre genérico), el Programa Remediar comprendió la provisión y distribución gratuita de medicamentos esenciales ambulatorios en los CAPS. Aunque estaba destinado a la población con cobertura de salud pública exclusiva, las rutinas y procedimientos no parecen haber impedido la entrega del medicamento a población con otra cobertura que acudía a ser atendida en los CAPS ${ }^{(26)}$.

Si bien sufrió distintos cambios, estuvo vigente durante todo el periodo de estudio bajo el nombre de Programa de Reforma de la Atención Primaria de la Salud II (PROAPS). En el año 2008, en el contexto del Ilamado Programa de Fortalecimiento de la Estrategia

Cuadro 1. Programa Remediar, Plan Nacer y Programa Sumar, según atributos básicos. Argentina, 2015.

\begin{tabular}{|c|c|c|c|c|c|c|c|c|}
\hline \multirow{2}{*}{$\begin{array}{l}\text { Programa / } \\
\text { Plan }\end{array}$} & \multirow{2}{*}{$\begin{array}{l}\text { Año de } \\
\text { inicio }\end{array}$} & \multirow[t]{2}{*}{ Jurisdicción } & \multirow[t]{2}{*}{ Organismo } & \multirow{2}{*}{$\begin{array}{l}\text { Población } \\
\text { cubierta }\end{array}$} & \multicolumn{2}{|c|}{ Financiamiento } & \multicolumn{2}{|c|}{ Efectores } \\
\hline & & & & & Fuente & Monto (en USD) & Tipo & Cantidad \\
\hline $\begin{array}{l}\text { Programa } \\
\text { Remediar }\end{array}$ & 2002 & Nacional & $\begin{array}{l}\text { Ministerio de } \\
\text { Salud de la } \\
\text { Nación }\end{array}$ & 16.086 .023 & $\begin{array}{l}\text { Banco } \\
\text { Interamericano de } \\
\text { Desarrollo }\end{array}$ & 139.726.962 & $\begin{array}{l}\text { Centros de } \\
\text { Atención } \\
\text { Primaria } \\
\text { de la salud }\end{array}$ & 7.823 \\
\hline $\begin{array}{l}\text { Plan Nacer } \\
\text { y Programa } \\
\text { Sumar }\end{array}$ & 2003 & Nacional & $\begin{array}{l}\text { Ministerio de } \\
\text { Salud de la } \\
\text { Nación }\end{array}$ & 12.787 .232 & $\begin{array}{l}\text { Banco } \\
\text { Interamericano de } \\
\text { Reconstrucción y } \\
\text { Fomento (BIRF), } \\
\text { y presupuesto } \\
\text { nacional y } \\
\text { provincial }\end{array}$ & 234.880 .546 & $\begin{array}{l}\text { Hospitales } \\
\text { y Centros } \\
\text { de } \\
\text { Atención } \\
\text { Primaria } \\
\text { de la salud }\end{array}$ & 7.515 \\
\hline
\end{tabular}


de Atención Primaria de la Salud (FEAPS), el cambio más significativo en el Programa Remediar fue la incorporación de un componente cuyo objetivo fue la conformación de redes de servicios de salud orientadas a la captación temprana y seguimiento de patologías crónicas no transmisibles (hipertensión y diabetes). Además de la contraparte nacional, contó en casi todo el periodo con un financiamiento del Banco Interamericano de Desarrollo (BID) que alcanzó los 230 miIlones de dólares estadounidenses ${ }^{(27)}$.

Concentrando su esfuerzo en los CAPS, el Programa buscó fortalecer la capacidad resolutiva del primer nivel, promover la estrategia de APS y capacitar a los distintos actores que lo componen en terapéutica racional, en el cuidado de medicamentos y en el ciclo de gestión del medicamento.

Hacia el final del periodo, los denominados "botiquines" comprendían 74 medicamentos que alcanzaban a 7.823 establecimientos desde los que se cubría a 16 millones de usuarios identificados ${ }^{(28)}$.

El sistema logístico de distribución consolidó un "sistema federal de abastecimiento centralizado" de medicamentos e insumos que permitía al resto de los programas del Ministerio de Salud de la Nación llegar a los CAPS de todo el país. A pesar de su centralización, la gestión comprometió, de distinto modo, tanto a provincias como a municipios, especialmente cuando éstos tenían a su cargo servicios del primer nivel.

Contaba con rigurosos y sistemáticos mecanismos de auditoría, monitoreo y evaluación. La gratuidad en la atención en la consulta era uno de los requisitos que el programa establecía para permanecer como efector. Las auditorías jugaron un papel fundamental, a partir de sus resultados se celebraban compromisos entre la nación, la provincia y los municipios orientados a corregir los problemas encontrados.

\section{Plan Nacer y Programa Sumar}

El Plan Nacer, posteriormente Ilamado Programa Sumar, fue un dispositivo de la política orientado a la conformación de seguros públicos provinciales destinados a garantizar un conjunto de prestaciones a la población sin cobertura explícita en salud. Contó con financiamiento del Banco Interamericano de Reconstrucción y Fomento (BIRF) y acumuló, desde 2005 (año en que se realizaron las primeras transferencias a las provincias) la suma de 234 millones de dólares estadounidenses (en transferencias hacia CAPS, hospitales y, aunque muy marginalmente, hacia otros prestadores privados). Estos aportes representaban el $1 \%$ de los recursos que invertían provincias y municipios en salud ${ }^{(29)}$, aunque con la potencia operativa de estar destinados de manera directa a los establecimientos para realizar erogaciones decididas por sus equipos.

En sus orígenes, el Plan Nacer buscó revertir el aumento de las tasas de morbimortalidad materna e infantil y proveer garantías explícitas para la población vulnerable, dirigiéndose a embarazadas y niños y niñas menores de 5 años.

Orientado originalmente al grupo materno infantil, en el año 2003 fue avanzando desde las provincias del norte del país hasta lograr una cobertura nacional en el año 2007. A partir del año 2011 se convirtió en el Programa Sumar, que amplió progresivamente la cobertura a otros grupos poblacionales: niños y niñas entre 6 y 9 años, adolescentes entre 10 y 19 años, mujeres y hombres hasta 64 años.

Al finalizar el periodo bajo análisis, el Programa Sumar buscaba alcanzar los siguientes objetivos: continuar disminuyendo las tasas de mortalidad materna e infantil; profundizar el cuidado de la salud de los niños y niñas en toda la etapa escolar y durante la adolescencia; mejorar el cuidado integral de la salud tanto de la mujer, promoviendo controles preventivos y buscando reducir la muerte por cáncer de útero y de mama; como del hombre, promoviendo controles preventivos y buscando reducir la muerte por cáncer colorrectal; crear y desarrollar los seguros de salud provinciales, destinados a la población más vulnerable ${ }^{(30)}$.

Se trataba de un sistema de incentivos monetarios asociados a resultados. Distintos instrumentos vinculaban las transferencias 
monetarias (a los seguros provinciales $y$, posteriormente, a los efectores) al hecho de haber alcanzado determinados resultados en cobertura poblacional, prestaciones a cubrir y metas sanitarias. Los mecanismos de auditoría, monitoreo y evaluación cumplieron un papel sustantivo para vincular las transferencias a los resultados sanitarios. En el marco de esos compromisos, cada efector recibía recursos financieros que podían destinar a la construcción y mejora de los edificios, a la compra y mantenimiento de equipamiento, a la contratación y capacitación de recursos humanos o a la compra de insumos no provistos por otros planes ${ }^{(31)}$.

Las metas sanitarias denominadas "trazadoras" eran un conjunto de indicadores destinados a medir distintos aspectos del proceso de atención sobre los que buscaba garantizar de manera explícita las prestaciones presentes en su nomenclador para toda la población, independientemente de la jurisdicción o dependencia del prestador a cargo ${ }^{(32)}$.

\section{Desafíos de la implementación en los escenarios subnacionales}

Retomando el enfoque del neoinstitucionalismo histórico, dos atributos permiten caracterizar la matriz institucional en Argentina por la que discurre la política orientada a la universalidad: el federalismo y la descentralización ${ }^{(26)}$.

El federalismo define el alcance y las limitaciones de las potestades de cada jurisdicción, en tanto significa que más de un gobierno regula un mismo territorio. Aunque en Argentina la salud no fue delegada por las provincias al gobierno federal, la Constitución Nacional reformada en el año 1994 consagra la garantía del derecho a la salud y sostiene, a su vez, su ejercicio bajo la órbita de las provincias, que siguen siendo las responsables de definir su alcance y organizar los modos de provisión de servicios bajo su órbita.

Sobre estas condiciones constitucionales, los procesos de descentralización convergieron también en el modelado de la red de hospitales y centros de salud. Los estudios sobre los procesos de descentralización muestran que fueron motivados más por el imperativo de resolver el conflicto financiero entre la nación y los estados provinciales, que por el interés de acercar los servicios a las preferencias de la población. Esta singularidad explica que las transferencias se hayan realizado sin atender a la articulación y a las necesidades de la organización de los servicios de salud y ayuda a comprender también sus consecuencias: la emergencia de gobiernos provinciales limitados en su capacidad de acción y con serios problemas en el financiamiento ${ }^{(36)}$.

Conviviendo con estas limitaciones, los estados provinciales ( $\mathrm{y}$, más tardíamente, también los municipios) fueron asumiendo la provisión de servicios de salud como parte de la agenda de sus gobiernos: crearon establecimientos, ampliaron servicios, innovaron en esquemas de organización y asumieron la gestión de hospitales y centros de salud. Estas apuestas fueron contemporáneas con el progresivo y radical retroceso del Estado nacional respecto del desarrollo de la oferta pública de salud. En ese devenir se fueron definiendo perfiles prestacionales, modalidades de acceso y modos de articulación con la seguridad social que variaron de una provincia a otra y hasta de un municipio a otro.

Estos procesos dieron lugar a escenarios provinciales y también municipales sumamente heterogéneos y también muy desiguales en su capacidad de respuesta financiera. Estas condiciones tuvieron una importancia capital para la configuración de los escenarios provinciales en los que discurren los esfuerzos de los programas nacionales orientados hacia la universalización en salud.

En los orígenes del Programa Remediar y del entonces denominado Plan Nacer, las provincias tenían a su cargo un $68 \%$ de los centros de salud (primer nivel) y un $73 \%$ de los hospitales (segundo nivel); mientras que casi la totalidad de los establecimientos restantes estaban bajo la órbita municipal $(30 \%$ de los centros de salud y $24 \%$ de los hospitales) y solo algunos pocos permanecían bajo la jurisdicción nacional. Esta división jurisdiccional sobre los efectores se expresaba 
también en la distribución de la inversión estatal en atención de la salud que, sin incluir los aportes a la seguridad social en sus distintas formas, presentaba los siguientes porcentajes: mientras que la realizada por el Estado nacional representaba el $0,33 \%$ del producto interno bruto (PIB), la inversión de las provincias era del $1,26 \%$ y la de los municipios del $0,26 \%$.

La distribución de responsabilidades heredada de la descentralización marcó también los márgenes de acción en cada nivel de gobierno en el comienzo de la década: mientras el Ministerio de Salud de la Nación (con apenas pocos establecimientos a cargo) logró liderar una agenda intergubernamental centrada en la estrategia de APS, las decisiones provinciales y también municipales estuvieron amarradas al imperativo de garantizar la provisión de servicios de salud en hospitales y centros de salud a su cargo.

Así, el "regreso" del Ministerio nacional a la arena de los servicios que representaron el Programa Remediar, el Plan Nacer y el Programa Sumar encontró 24 escenarios provinciales sumamente heterogéneos, tanto en el modo en que distribuían las funciones con relación a la provisión de servicios, como en las orientaciones que animaban a la política que (no siempre de manera progresiva) incidían en las condiciones a través de las cuales se avanzaba hacia la universalización de la salud.

La jerarquización del primer nivel asistencial por parte de la política nacional enfrentó al Ministerio de Salud de la Nación con dos niveles diferentes de interlocución que variaban en su responsabilidad sobre la provisión de servicios: los estados provinciales y los municipios. Las iniciativas nacionales mostraron capacidad para definir un papel más claro (aunque no siempre coincidente en el interior de la política nacional) para las provincias y un lugar menos explícito para los municipios, cuya participación en la provisión de servicios estatales variaba de una provincia a otra.

La provincia fue un punto de veto para el desarrollo de la política nacional no solo por las características de la organización federal argentina, sino porque allí radicaba y radica la potestad sanitaria de la cual derivan funciones relativas a la interlocución institucional con el contexto, a la gestión de los recursos y a la sanción de normas orientadas a los procesos de atención. La "adhesión a leyes" y la celebración de "convenios de adhesión" institucionalizaron en el periodo analizado la aceptación de las políticas nacionales por los estados provinciales y establecieron los compromisos de cada parte.

\section{Avances hacia el universalismo}

Los dispositivos de la política analizados -Programa Remediar, Plan Nacer y Programa Sumar- muestran distintos intentos de cambio en las variables bajo análisis: acceso, cobertura, beneficios y derechos.

\section{Acceso}

La apuesta de la política pública nacional por el fortalecimiento del primer nivel de atención fue una estrategia presente en los dispositivos analizados en todo el periodo, que buscó facilitar la obtención de atención sanitaria por parte de la población de mayor vulnerabilidad. Según una caracterización de la demanda realizada por el Programa Remediar, el $82 \%$ de los consultantes a los CAPS se concentraban en el quintil 1 y 2 de ingresos y 7 de cada 10 tenía cobertura exclusiva del subsector estatal( ${ }^{(28)}$.

Esta estrategia encontró una trama vasta y heterogénea de centros de salud y postas sanitarias provinciales y municipales que presentaba distintas formas de mercantilización en el acceso a los servicios, entre las cuales el cobro del "bono" contribución era la modalidad más extendida. En este contexto, el Programa Remediar buscó librar la batalla por la remoción de las barreras económicas en el acceso a través de formalizar la gratuidad de la atención como requisito para mantener la implementación del programa en cada CAPS, mecanismo fortalecido por las auditorías concurrentes y la discusión de sus informes en los encuentros tripartitos nación, provincia 
y municipios. Un estudio realizado sobre 18.317 auditorías detectó la presencia de cobro en la atención (consultas profesionales o prácticas médicas), ganando incidencia el cobro obligatorio en las consultas a expensas del optativo, con diferencias muy importantes entre provincias y -dentro de estas- entre municipios. Si bien este estudio muestra un recorrido errático a lo largo del periodo, la Encuesta de Utilización y Gastos en Servicios de Salud evidencia logros expresados en la reducción de la proporción de la población que enfrentaba barreras financieras en las consultas en los servicios del subsector estatal, del $13 \%$ en el año 2003 al $5 \%$ en el año $2010^{(33)}$.

El resguardo de la gratuidad en las prestaciones fue también un requisito para permanecer como "proveedor" del Plan Nacer, posteriormente Ilamado Programa Sumar, aunque en este caso se destaca el énfasis puesto en la aplicación de incentivos financieros para promover el acceso oportuno a determinadas prestaciones (control del niño sano, trimestre en el control del embarazo, entre otras).

\section{Cobertura}

Con relación a la segunda variable de análisis, asegurar la cobertura de servicios a la población fue otra de las preocupaciones de la política nacional, especialmente, en un contexto que mostraba resistencias para reducir el porcentaje de población que no llegaba a estar cubierta por la seguridad social o por seguros privados (31\% en el año 2011) ${ }^{(38)}$.

En ese marco, tanto el Programa Remediar como el Plan Nacer y el Programa Sumar nominalizaron a la población para concentrar el esfuerzo en aquellos para quienes los servicios de salud estatales eran la única alternativa. En el Plan Sumar, rigurosos mecanismos asociaban los instrumentos de pago al cumplimiento del requisito de focalización (población que no estuviera cubierta por obra social o seguro privado). Como resultado, en el año 2015 el $100 \%$ de los 12.787 .232 millones de personas cubiertas por el programa $^{(35)}$ no poseían otra cobertura que la que brindaba el Estado.
A pesar de compartir criterios formales idénticos con relación a la definición de su población objetivo, el $66 \%$ de los consultantes del Programa Remediar tenía, en el año 2013, al Estado como único proveedor de servicios de salud, mientras que el $82 \%$ del total pertenecía a los dos quintiles más bajos de ingresos ${ }^{(39)}$.

La Asignación Universal por Hijo (AUH), un seguro social que incorporó al Régimen de Asignaciones Familiares a los niños, adolescentes y jóvenes -hasta los 18 años-, independientemente de la condición que revistieran sus padres en el mercado de trabajo, sostuvo la condicionalidad de realización de controles de salud para hacer efectivo un porcentaje de la asignación con impactos diferenciales según los programas bajo análisis. Con el Plan Nacer se incrementó un 50\% la inscripción de niños y niñas y creció un $12 \%$ el número de niños y niñas con controles completos; también se dieron procesos inversos: 230 mil niños y niñas ingresaron a la AUH por estar inscriptos en el Plan Nacer ${ }^{(40)}$. En el Programa Remediar los resultados en materia de integración parecen haber sido menores: de los 5.096.267 niños entre 0 y 18 años beneficiarios del Programa Remediar, el $19 \%$ (991.745) percibe la $A \cup H$, mientras que el $44 \%$ es beneficiario del Programa Sumar. Este porcentaje se incrementa al $80 \%$ cuando se consideran a los menores de 6 años ${ }^{(28)}$.

\section{Beneficios}

La heterogeneidad de respuestas del Estado a nivel provincial y municipal llevó a la política nacional a plantearse también la necesidad de garantizar de manera explícita un conjunto homogéneo de beneficios en cantidad, calidad y oportunidad para toda la población. La capacitación de los equipos de salud, la regulación de las prescripciones y la provisión de medicamentos esenciales en el primer nivel (realizadas a través del Programa Remediar), como así también la adopción de un "nomenclador de prestaciones" y las metas "trazadoras" asociadas a los mecanismos de pago a los prestadores del Plan Nacer y el Programa Sumar, son las herramientas que 
utilizaron los dispositivos bajo análisis para alcanzar estándares homogéneos en las prestaciones, independientemente de la jurisdicción de los centros de salud y hospitales.

Los medicamentos provistos por el Programa Remediar representaron el 85\% de los medicamentos entregados de forma gratuita en efectores del primer nivel de atención. Un estudio cuantitativo de impacto sobre la equidad realizado en el año 2013 mostró que la transferencia del Programa Remediar redujo el gasto total en medicamentos de las personas de los quintiles de ingreso más bajos ${ }^{(28)}$.

Al tiempo que fueron ampliando su población objetivo, el Plan Nacer y el Programa Sumar incorporaron nuevas prestaciones. Comenzaron en 2005, con un plan de beneficios básicos orientados a la población materno-infantil -en su mayoría preventivos y de promoción de la salud-, provistos por establecimientos de baja complejidad. El alcance aumentó gradualmente $y$, a partir del año 2010, incorporaron el cuidado de otros grupos de edad y la ampliación al cuidado integral de cardiopatías congénitas, prestaciones de embarazo de alto riesgo y servicios de neonatología (incluyendo tratamiento, diagnóstico y los medicamentos requeridos). A partir del año 2015, el programa incorporó la prevención secundaria de enfermedades crónicas no transmisibles en hombres de 20 a 64 años.

El Plan Nacer y el Programa Sumar definieron un conjunto de prestaciones priorizadas, consideradas esenciales para el buen cuidado de la salud, que formaban parte del nomenclador y podían ser facturadas a cada Seguro Provincial de Salud. Se trata de 400 prestaciones de prevención primaria y promoción de la salud, y actividades de tratamiento y prevención secundaria organizadas en 47 líneas de cuidado que conformaban un nomenclador único y homogéneo para todo el país que buscaba precisar atributos de calidad requeridos para cada una de ellas ${ }^{(29)}$.

\section{Derechos}

A pesar de la relevancia que tuvieron para el sector y de los desafíos que enfrentaron en una matriz institucional muy fragmentada, los dispositivos de la política analizados no estuvieron acompañados de arreglos institucionales que, en el nivel nacional, implicaran alguna exigencia vinculada a la ampliación de derechos.

El Programa Remediar buscó integrarse a la estructura organizativa del Ministerio de Salud, mientras que el Plan Nacer y el Programa Sumar fueron gestionados desde una unidad ejecutora. Sin embargo, en el nivel provincial la conformación de "seguros de salud" y los compromisos presupuestarios que los acompañaban son avances cuya efectividad, de cara al universalismo, deberá ser evaluada en el futuro.

La cobertura condicionada a la no disposición de obra social y los instrumentos de pago utilizados por el Plan Nacer y el Programa Sumar ponen en evidencia no solo una preocupación por garantizar la cobertura de toda la población, sino también el interés por promover la separación de funciones entre financiadores y proveedores para coordinar el financiamiento entre niveles de gobierno. Con relación a esta última orientación, en los caminos hacia la universalización propuestos desde la política nacional hasta el año 2015, coexistieron dos modelos sanitarios: uno perfilado hacia la construcción de un seguro público (representado por el Plan Nacer y el Programa Sumar) y otro orientado hacia el fortalecimiento de la oferta pública (Programa Remediar). Aunque reconocen un mismo origen (el Plan Federal de Salud 2004-2007), estos programas ponen en evidencia las diferencias existentes al interior del Ministerio de Salud de la Nación en los modos de concebir la recuperación de la capacidad de rectoría sobre una red de establecimientos estatales que, hacía varias décadas, ocupaba la preocupación de otras instancias de gobierno. 


\section{CONCLUSIONES}

En términos generales, la acción de la política nacional se concentró sobre las relaciones entre la población y los proveedores, buscando regular y fortalecer aspectos críticos de la práctica médica e institucional en el nivel de los servicios. Para esto se movilizaron distintos incentivos monetarios y no monetarios (medicamentos, equipamiento médico y no médico, incentivos por producción, entre otros) que se dirigieron directamente a los CAPS y, con menor intensidad, a los hospitales estatales de provincias y municipios. A su vez, las estrategias adoptadas por casi la totalidad de los dispositivos estuvieron dirigidas hacia la capacitación de los equipos de salud y la elaboración de normas para la atención.

En la implementación -y cumpliendo con los requisitos que el federalismo en salud impone a la implementación de los programas nacionales- cada uno de los dispositivos bajo análisis confirió al Estado provincial un lugar y márgenes de autonomía diferentes con relación al modo de asignar los recursos. Aunque el Programa Remediar, el Plan Nacer y el Programa Sumar convergieron en la idea de construir una relación directa con los CAPS, el primero lo hizo buscando fortalecer la oferta de servicios estatales, mientras que el segundo procuró establecer una separación de funciones entre financiamiento y provisión.

Los "modelos de cambio" implícitos en los dispositivos de políticas analizados, supusieron jerarquizar de modo diferente la instancia provincial: en el Programa Remediar en la medida que fuera responsable de los CAPS; en el Plan Nacer y el Programa Sumar la instancia provincial representó el espacio para conformar seguros públicos provinciales, como compradores de servicios a los prestadores (tanto públicos como privados). En aquellos escenarios en los que la provisión de servicios estuvo a cargo (total o parcialmente) de los municipios, las distinciones aparecieron en el lugar asignado a este nivel de gobierno: mientras el Programa Remediar le confirió el papel de "responsable de la provisión de los servicios" y demarcó su lugar a través de sus rutinas organizacionales, el Plan Nacer y el Programa Sumar lo ubicaron en el lugar de "administrador de fondos de terceros" de los CAPS (definidos como "proveedores público-privados") e insistió en la soberanía de estos para la asignación de los recursos derivados de la facturación de prestaciones $^{(24)}$.

Las iniciativas nacionales analizadas instituyeron (en sus reglas de juego y en sus rutinas) un nuevo actor, el equipo del CAPS, con márgenes de autonomía diferentes en cada dispositivo según el aspecto de la práctica médica y/o de la rutina organizacional que buscó regular.

A la vez, ambos dispositivos abonaron al fortalecimiento de la capacidad de rectoría del Ministerio de Salud de la Nación, aunque desde distintas perspectivas. El Programa Remediar tuvo un papel fundante en tanto se trató de una política centralizada de distribución de medicamentos con fuertes mecanismos de auditoría, monitoreo y evaluación, aunque en la gestión también comprometió a provincias y municipios con establecimientos de primer nivel a su cargo. El Plan Nacer y el Programa Sumar apostaron, en cambio, a mantener la capacidad de rectoría con relación a los problemas de cobertura y garantía de un conjunto de prestaciones en cantidad y calidad, dejando abiertos distintos márgenes de autonomía para la decisión provincial.

En el proceso de universalización, la política nacional se orientó hacia el fortalecimiento del primer nivel de atención para promover el acceso, estrategia que estuvo presente en los dispositivos analizados, al facilitar la obtención de atención sanitaria gratuita a la población sin otra cobertura que la estatal. Así, el Programa Remediar buscó remover barreras económicas como condición para entregar los botiquines a los CAPS y el Plan Nacer y el Programa Sumar resguardaron la gratuidad de las prestaciones al imponerla como requisito para permanecer como proveedor del programa.

La cobertura de servicios a la población fue otra de las preocupaciones, en especial, 
en un contexto que mostraba resistencias para reducir el porcentaje de población que no llegaba a estar cubierta por la seguridad social o por seguros privados. Tanto el Programa Remediar como el Plan Nacer y el Programa Sumar nominalizaron a la población, y concentraron el esfuerzo en aquellos que tenían los servicios de salud estatales como única alternativa.

Con relación a los beneficios, la heterogeneidad de respuestas del Estado a nivel provincial y municipal, llevó a plantear también la necesidad de garantizar de manera explícita un conjunto homogéneo de prestaciones en cantidad, calidad y oportunidad para toda la población. La capacitación de los equipos de salud, la regulación de las prescripciones y la provisión de medicamentos esenciales en el primer nivel realizadas a través del Programa Remediar, como así también la adopción de un "nomenclador de prestaciones" y las metas "trazadoras" asociadas a los mecanismos de pago a los prestadores del Plan Nacer y el Programa Sumar fueron las herramientas utilizadas para alcanzar estándares homogéneos en las prestaciones, independientemente de la jurisdicción de los centros de salud y hospitales.

En el contexto de la matriz institucional argentina, estas orientaciones programáticas libraron sus batallas en espacios de implementación configurados de manera diversa (en los perfiles prestacionales, las modalidades de acceso y los modos de articulación con la seguridad social), abriendo para la investigación distintas preguntas acerca de los caminos hacia la universalidad a ser respondidas en el nivel subnacional y a través de estudios delimitados a contextos territoriales e institucionales específicos.

\section{AGRADECIMIENTOS}

Este trabajo es resultado de la investigación "Cobertura universal de salud y redes de servicios: encuentros y desencuentros entre la macro y la meso gestión. Un estudio de caso en el conurbano bonaerense 2008/2015" (Proyectos de Investigación Científica y Tecnológica (2014) PICT 0693 desarrollado en el Instituto del Conurbano de la Universidad Nacional de General Sarmiento, Buenos Aires, Argentina.

\section{REFRENCIAS BIBLIOGRÁFICAS}

1. Bergallo P. Argentina: los tribunales y el derecho a la salud. En: Yamin AE, Gloppen S. La lucha por los derechos de la salud: ¿Puede la justicia ser una herramienta de cambio? Buenos Aires: Siglo XXI Editores; 2013.

2. Laurell AC. Contradicciones en salud: sobre acumulación y legitimidad en los gobiernos neoliberales y sociales de derecho en América Latina. Saúde em Debate. 2014;38(103):853-871.

3. Abramovich V, Pautassi L. Dilemas actuales en la resolución de la pobreza: El aporte del enfoque de derechos. Seminario "Los Derechos Humanos y las Políticas Públicas para Enfrentar la Pobreza y la Desigualdad"; Universidad Nacional Tres de Febrero, Buenos Aires. Buenos Aires: UNESCO, Secretaría de Derechos Humanos; 2006.
4. Cetrángolo O. Aportes para el desarrollo humano en Argentina 2011. Buenos Aires: PNUD, OPS, CEPAL; 2011.

5. Cunill Grau N. Las políticas con enfoque de derechos y su incidencia en la institucionalidad pública. Revista del CLAD Reforma y Democracia. 2010;46:41-72.

6. Crojethovic M. Universalismo en salud: La reconstrucción de un debate sobre los derechos. Revista Vía luris. 2016;21:13-23.

7. Organización Mundial de la Salud. Hacia la cobertura sanitaria universal: conceptos, enseñanzas extraídas y desafíos para las políticas públicas. Revista Española de Salud Pública. 2013;8 (1):3-9.

8. Organización Mundial de la Salud. Estrategia para el acceso universal a la salud y la cobertura universal de salud [Internet]. Washington DC: OMS; 2014 [citado 25 ene 2017]. Disponible en: https://goo.gl/qMW7At.

9. Pressman J, Wildavsky A. Prefacio a la primera edición. En: Implementación: Cómo grandes expectativas concebidas en Washington se frustran en Oakland. México: Fondo de Cultura Económica; 1998.

10. Aguilar Villanueva LF. Estudio introductorio. En: La implementación de las políticas públicas. México: Miguel Ángel Porrúa; 1996. 
11. Belmartino S. Desarrollo metodológico para una historia comparada de la profesión médica (Documento de Trabajo No 80). Buenos Aires: Centro Interdisciplinario para el estudio de las políticas públicas; 2011.

12. March JG, Olsen JP. Las reglas y la institucionalización de la acción. En: El redescubrimiento de las instituciones: La base organizativa de la política. México: Fondo de Cultura Económica; 1997.

13. Pierson $\mathrm{P}$, Skocpol T. El institucionalismo histórico en la ciencia política contemporánea. Revista Uruguaya de Ciencia Política. 2008;17(1):7-38.

14. Hall P, Taylor R. Political science and the three new institutionalisms. Political Studies. 1996;XLIV:936-957.

15. Naciones Unidas para los Derechos Humanos. Estándares internacionales sobre el derecho a la salud en el sistema de Naciones Unidas. Colombia: Oficina del Alto Comisionado de las Naciones Unidas para los Derechos Humanos; 2015.

16. De Riz L. Argentina, una vez más en la encrucijada. Temas y Debates. 2008;(16):9-27.

17. Ministerio de Salud de la Nación, Organización Mundial de la Salud, Organización Panamericana de la Salud. Indicadores Básicos Argentina 2015 [Internet]. Argentina: Ministerio de Salud de la Nación, OMS, OPS; 2015 [citado 25 ene 2017]. Disponible en: https://tinyurl.com/ yaj $45 \mathrm{~m} 74$.

18. Banco Mundial. Indicadores del desarrollo mundial: PBI per cápita año 2015 (a precios actuales) [Internet]. Banco Mundial [citado 25 ene 2017]. Disponible en: https://tinyurl.com/ yd4hbu9j.

19. Instituto Nacional de Estadística y Censos. Censo Nacional de Población, Hogares y Viviendas 2010: Cuestionario Ampliado [Internet]. INDEC [citado 25 ene 2017]. Disponible en: https://tinyurl.com/ybc5ndvg.

20. Maceira D, Apella I, Barbieri E. Necesidades de la demanda y subsidios de medicamentos: La experiencia del Programa Remediar en Argentina. Buenos Aires: CEDES; 2005.

21. Cetrángolo O, Lima Quintana L, San Martín M. Situación del sector salud en Argentina: Análisis en el contexto de un sistema descentralizado. Buenos Aires: Banco Interamericano de Desarrollo; 2007.
22. Findling L, Champalbert L. La política de salud en Argentina: Avances y cuestiones pendientes. Ciencias Sociales, Revista de la Facultad de Ciencias Sociales. 2007;(69):30-31.

23. Arce H. El sistema de salud: De dónde viene y hacia dónde va. Buenos Aires: Prometeo; 2010.

24. Chiara M. Políticas e instituciones en salud: el desafío de construir nuevos escenarios. Debate Público, Reflexión de Trabajo Social. 2013;3(6):31-42.

25. Potenza dal Masetto F. La implementación del Plan Nacer en ámbitos subnacionales (Documento de Trabajo No. 82). Buenos Aires: CIPPEC, Programa de Protección Social, Área de Desarrollo Social; 2012.

26. Chiara M. Política de atención de la salud y relaciones intergubernamentales (RIG): continuidades e inflexiones en los patrones de interacción en el Gran Buenos Aires, Argentina (2001-2011). [Tesis de doctorado]. Buenos Aires: Facultad de Ciencias Sociales, Universidad de Buenos Aires; 2013.

27. Banco Interamericano de Desarrollo. Programa de Fortalecimiento de la Estrategia de Atención Primaria de la Salud [Internet]. 2006 [citado 25 ene 2017]. Disponible en: https://tinyurl.com/ y7bz8wm5.

28. Ministerio de Salud de la Nación, Programa Remediar. Acceso a medicamentos esenciales e impacto redistributivo. Buenos Aires: Ministerio de Salud de la Nación, Programa Remediar; 2015.

29. Sabignoso $M$, Silva $H$, Curcio J. Argentina: el Plan Nacer. En: Giedion U, Bitrán R, Tristao I. Planes de beneficios en salud de América Latina: una comparación regional. Buenos Aires: Banco Interamericano de Desarrollo, División de Protección Social y Salud; 2014.

30. Ministerio de Salud de la Nación. Programa Sumar: Plan de servicios de salud. Buenos Aires: Ministerio de Salud de la Nación; 2015.

31. Ministerio de Salud de la Nación. Plan Nacer: Manual Operativo. Buenos Aires: Ministerio de Salud de la Nación; 2008.

32. Giedion U, Bitrán R, Tristao I. Explicitar lo implícito: Análisis de siete planes de beneficios en salud de América. En: Giedion U, Bitrán R, Tristao I. Planes de beneficios en salud de América Latina: una comparación regional. Buenos Aires: Banco Interamericano de Desarrollo, División de Protección Social y Salud; 2014. 
33. Ballesteros M. Evolución y localización geográfica de barreras financieras para el acceso a servicios de salud en el primer nivel de atención del sector público. Buenos Aires: Ministerio de Salud de la Nación; 2015.

34. Ministerio de Salud de la Nación. ¿Quiénes consultan en los centros de salud, por qué motivos y qué se les prescribe? Un análisis de las recetas Remediar [Internet]. Buenos Aires: Ministerio de Salud de la Nación; marzo 2015 [citado 25 ene 2017]. Disponible en: https://tinyurl.com/ yd2zycz 7

35. Ministerio de Salud de la Nación. Reporte de gestión Sumar [Internet]. Buenos Aires: Ministerio de Salud de la Nación; 2015 [citado 25 ene 2017]. Disponible en: https://tinyurl.com/yasdlzdx.

36. Centrángolo $\mathrm{O}$, Jiménez JP. Las relaciones entre niveles de gobierno en Argentina: Raíces históricas, instituciones y conflictos persistentes. Santiago de Chile: ILPES, CEPAL; 2004. (Serie Gestión Pública No. 47).
37. Dirección de Análisis de Gasto Público y Programas Sociales, Secretaría de Política Económica, Ministerio de Economía de la Nación. Serie Gasto público nacional, provincial y municipal consolidado [Internet]. Buenos Aires: Ministerio de Economía de la Nación [citado 25 ene 2017]. Disponible en: https://tinyurl.com/yd4dg8mr.

38. Instituto Nacional de Estadísticas y Censos. Encuesta Permanente de Hogares. Buenos Aires: INDEC; 2011.

39. Ministerio de Salud de la Nación. Evaluación y monitoreo 2013: Acceso a medicamentos, diversas miradas, un mismo derecho [Internet]. Buenos Aires: Ministerio de Salud de la Nación; 2013 [citado 25 ene 2017]. Disponible en: https:// tinyurl.com/yazxkq6n.

40. Ministerio de Salud de la Nación. Programa Sumar, la ampliación del Plan Nacer [Internet]. Buenos Aires: Ministerio de Salud de la Nación; 2011 [citado 25 ene 2017] https://tinyurl.com/ ybuumjj4.

\section{FORMA DE CITAR}

Chiara M, Crojethovic M, Ariovich A. El universalismo en salud en Argentina entre 2003 y 2015: balances y desafíos desde una aproximación macro institucional. Salud Colectiva. 2017;13(4):577-597. doi: 10.18294/sc.2017.1312

Recibido: 2 de febrero de 2017 | Versión final: 5 de mayo de 2017 | Aprobado: 30 de mayo de 2017 